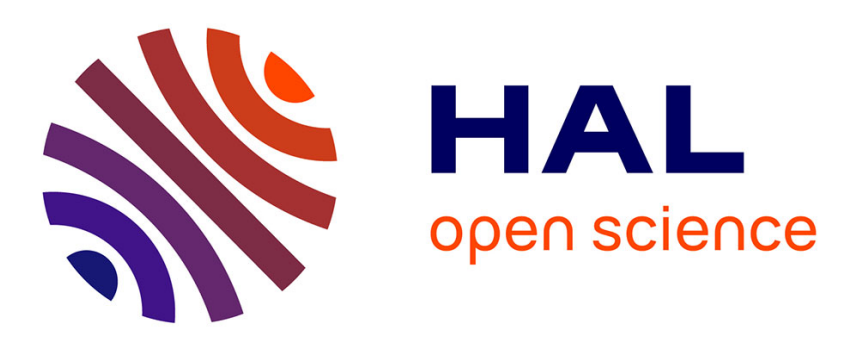

\title{
Multichannel HR-NMF for modelling convolutive mixtures of non-stationary signals in the time-frequency domain
}

\author{
Roland Badeau, Mark. D. Plumbley
}

\section{- To cite this version:}

Roland Badeau, Mark. D. Plumbley. Multichannel HR-NMF for modelling convolutive mixtures of non-stationary signals in the time-frequency domain. WASPAA, 2013, New Paltz, New York, United States. hal-00945278

\section{HAL Id: hal-00945278 \\ https://hal.inria.fr/hal-00945278}

Submitted on 25 Mar 2014

HAL is a multi-disciplinary open access archive for the deposit and dissemination of scientific research documents, whether they are published or not. The documents may come from teaching and research institutions in France or abroad, or from public or private research centers.
L'archive ouverte pluridisciplinaire HAL, est destinée au dépôt et à la diffusion de documents scientifiques de niveau recherche, publiés ou non, émanant des établissements d'enseignement et de recherche français ou étrangers, des laboratoires publics ou privés. 


\section{MULTICHANNEL HR-NMF FOR MODELLING CONVOLUTIVE MIXTURES OF NON-STATIONARY SIGNALS IN THE TIME-FREQUENCY DOMAIN}

\author{
Roland Badeau* \\ Institut Mines-Telecom \\ Telecom ParisTech \\ CNRS LTCI
}

\author{
Mark D. Plumbley ${ }^{\dagger}$ \\ Centre for Digital Music, School of \\ Electronical Engineering \& Computer Science \\ Queen Mary University of London
}

\begin{abstract}
In the literature, several probabilistic models involving latent components have been proposed for modelling time-frequency (TF) representations of audio signals (such as spectrograms), notably in the nonnegative matrix factorization (NMF) literature. Among them, the recent high resolution (HR)-NMF model is able to take both phases and local correlations in each frequency band into account, and its potential has been illustrated in applications such as source separation and audio inpainting. In this paper, HR-NMF is extended to multichannel signals and to convolutive mixtures. A fast variational expectation-maximization (EM) algorithm is proposed to estimate the enhanced model. This algorithm is applied to a stereophonic piano signal, and proves capable of accurately modelling reverberation and restoring missing observations.
\end{abstract}

Index Terms - Non-stationary signal modelling, Timefrequency analysis, Separation of convolutive mixtures, Multichannel signal analysis, Variational EM algorithm.

\section{INTRODUCTION}

Following previous works which aimed to provide a probabilistic framework to NMF [1-4], the HR-NMF model introduced in [5,6] offers an improved frequency resolution (it can separate sinusoids within the same frequency band) and an improved synthesis capability (it can restore missing TF observations). It is suitable for both complex-valued and real-valued TF representations, such as the short-time Fourier transform (STFT) and the modified discrete cosine transform (MDCT). It also generalizes some popular models, such as the Itakura-Saito NMF model (IS-NMF) [4], autoregressive (AR) processes [7], and the exponential sinusoidal model (ESM), commonly used in HR spectral analysis of time series [7].

In this paper, HR-NMF is extended to multichannel signals and to convolutive mixtures. Contrary to the multichannel NMF [8] where convolution was approximated, convolution is here accurately modelled in the TF domain by following the exact implementation proposed in [9]. Consequently, correlations within and between frequency bands are both taken into account. In order to estimate this multichannel HR-NMF model, we propose a variational EM algorithm, whose novelties compared to [10] include a reduced computational complexity and a parallel implementation.

${ }^{*}$ This work was undertaken while Roland Badeau was visiting the Centre for Digital Music, partly funded by EPSRC Platform Grant EP/K009559/1, and by the French National Research Agency (ANR) as a part of the DReaM project (ANR-09-CORD-006-03).

${ }^{\dagger}$ Mark D. Plumbley is funded by EPSRC Leadership Fellowship $\mathrm{EP} / \mathrm{G} 007144 / 1$.
The paper is structured as follows. The multichannel HR-NMF model is introduced in section 2, and the variational EM algorithm is derived in section 3. An application to a stereophonic piano signal is presented in section 4. Finally, conclusions are drawn in section 5.

\section{MULTICHANNEL HR-NMF MODEL}

The multichannel HR-NMF model of TF data $y_{m}(f, t) \in \mathbb{F}$ (where $\mathbb{F}=\mathbb{R}$ or $\mathbb{C})$ is defined for all channels $m \in[0 \ldots M-1]$, discrete frequencies $f \in[0 \ldots F-1]$, and times $t \in[0 \ldots T-1]$, as the sum of $S$ components $y_{m s}(f, t) \in \mathbb{F}$ plus a white noise $n_{m}(f, t) \sim$ $\mathcal{N}_{\mathbb{F}}\left(0, \sigma_{y}^{2}\right)$, where $\mathcal{N}_{\mathbb{F}}\left(0, \sigma_{y}^{2}\right)$ denotes a real (if $\mathbb{F}=\mathbb{R}$ ) or circular complex (if $\mathbb{F}=\mathbb{C}$ ) normal distribution of mean 0 and variance $\sigma_{y}^{2}$ :

$$
y_{m}(f, t)=n_{m}(f, t)+\sum_{s=0}^{S-1} y_{m s}(f, t) .
$$

Each component $y_{m s}(f, t)$ for any $s \in[0 \ldots S-1]$ is defined as

$$
y_{m s}(f, t)=\sum_{\varphi=-P_{b}}^{P_{b}} \sum_{\tau=0}^{Q_{b}} b_{m s}(f, \varphi, \tau) z_{s}(f-\varphi, t-\tau)
$$

where $b_{m s}(f, \varphi, \tau)=0$ if $f-\varphi \notin[0 \ldots F-1]$, and the latent component $z_{s}(f, t)$ is defined as follows:

- $\forall t \in[0 \ldots T-1], x_{s}(f, t) \sim \mathcal{N}_{\mathbb{F}}\left(0, \sigma_{x_{s}}^{2}(t)\right)$ and

$$
z_{s}(f, t)=x_{s}(f, t)-\sum_{\tau=1}^{Q_{a}} a_{s}(f, \tau) z_{s}(f, t-\tau)
$$

where $a_{s}(f, \tau)$ defines a causal and stable autoregressive filter,

- $\forall t \in\left[-Q_{z} \ldots-1\right]$ where $Q_{z}=\max \left(Q_{b}, Q_{a}\right), z_{s}(f, t) \sim$ $\mathcal{N}\left(\mu_{s}(f, t), 1 / \rho_{s}(f, t)\right)$.

Moreover, all scalar random variables $n_{m}(f, t)$ and $x_{s}(f, t)$ are assumed independent. Besides, $\forall m \in[0 \ldots M-1], \forall f \in$ $[0 \ldots F-1], \forall t \in\left[-Q_{z} \ldots-1\right], y_{m}(f, t)$ is unobserved, and $\forall s \in[0 \ldots S-1]$, the prior mean $\mu_{s}(f, t)$ and the prior precision (inverse variance) $\rho_{s}(f, t)$ of the latent variable $z_{s}(f, t)$ are considered as known (fixed) parameters.

The set $\theta$ of parameters to be estimated consists of:

- the autoregressive parameters $a_{s}(f, \tau) \in \mathbb{F}$ for $s \in$ $[0 \ldots S-1], \tau \in\left[1 \ldots Q_{a}\right]$ and $f \in[0 \ldots F-1]$ (we further define $\left.a_{s}(f, 0)=1\right)$,

- the moving average parameters $b_{m s}(f, \varphi, \tau) \in \mathbb{F}$ for $m \in$ $[0 \ldots M-1], s \in[0 \ldots S-1], f \in[0 \ldots F-1], \varphi \in$ $\left[-P_{b} \ldots P_{b}\right]$, and $\tau \in\left[0 \ldots Q_{b}\right]$, 
- the variance parameters $\sigma_{y}^{2}>0$ and $\sigma_{x_{s}}^{2}(t)>0$ for $s \in$ $[0 \ldots S-1]$ and $t \in[0 \ldots T-1]$.

This model encompasses the following particular cases:

- If $M=1, \sigma_{y}^{2}=0$ and $P_{b}=Q_{b}=Q_{a}=0$, equation (1) becomes $y_{0}(f, t)=\sum_{s=0}^{S-1} b_{0 s}(f, 0,0) x_{s}(f, t)$, thus $y_{0}(f, t) \sim$ $\mathcal{N}_{\mathbb{F}}\left(0, \widehat{V}_{f t}\right)$, where $\widehat{\boldsymbol{V}}$ is defined by the NMF $\widehat{\boldsymbol{V}}=\boldsymbol{W} \boldsymbol{H}$ with $W_{f s}=\left|b_{0 s}(f, 0,0)\right|^{2}$ and $H_{s t}=\sigma_{x_{s}}^{2}(t)$. The maximum likelihood estimation of $\boldsymbol{W}$ and $\boldsymbol{H}$ is then equivalent to the minimization of the Itakura-Saito (IS) divergence between matrix $\widehat{\boldsymbol{V}}$ and spectrogram $\boldsymbol{V}$ (where $V_{f t}=\left|y_{0}(f, t)\right|^{2}$ ), hence this model is referred to as IS-NMF [4].

- If $M=1$ and $P_{b}=Q_{b}=0, y_{0}(f, t)$ follows the HR-NMF model $[5,6,10]$ involving variance $\sigma_{y}^{2}$, autoregressive parameters $a_{s}(f, \tau)$, and same NMF $\widehat{\boldsymbol{V}}=\boldsymbol{W} \boldsymbol{H}$.

- If $S=1, \sigma_{y}^{2}=0, P_{b}=0, \forall t \in[0 \ldots T-1], \sigma_{x_{0}}^{2}(t)=1$, and $\forall t \in\left[-Q_{z} \ldots-1\right], \mu_{s}(f, t)=0$ and $\left.\rho_{s}(f, t)\right)=1$, then $\forall m \in[0 \ldots M-1], \forall f \in[0 \ldots F-1], y_{m}(f, t)$ is an autoregressive moving average (ARMA) process [7].

- If $S=1, \sigma_{y}^{2}=0, P_{b}=0, Q_{a}>0, Q_{b}=Q_{a}-1$, $\forall t \in\left[-Q_{z} \ldots-1\right], \mu_{0}(f, t)=0, \rho_{0}(f, t) \rightarrow+\infty$, and $\sigma_{0}^{2}(t)=\mathbb{1}_{\{t=0\}}$, then $\forall m \in[0 \ldots M-1], \forall f \in$ $[0 \ldots F-1], y_{m}(f, t)$ can be written in the form $y_{m}(f, t)=$ $\sum_{\tau=1}^{Q_{a}} \alpha_{m \tau} z_{\tau}^{t}$, where $z_{1} \ldots z_{Q_{a}}$ are the roots of the polynomial $z^{Q_{a}}-\sum_{\tau=1}^{Q_{a}} a_{s}(f, \tau) z^{Q_{a}-\tau}$. This corresponds to the Exponential Sinusoidal Model (ESM) commonly used in HR spectral analysis of time series [7].

\section{VARIATIONAL EM ALGORITHM}

In order to estimate the multichannel HR-NMF model introduced in section 2, we derive below a variational EM algorithm.

\subsection{Review of variational EM algorithm}

Variational inference [11] is now a classical approach for estimating a probabilistic model involving both observed variables $y$ and latent variables $z$, parametrized by $\theta$. Let $\mathcal{F}$ be a set of probability density functions (PDF) over the latent variables $z$. For any PDF $q \in \mathcal{F}$ and any function $f(z)$, we note $\langle f\rangle_{q}=\int f(z) q(z) d z$. Then for any parameter $\theta$, the variational free energy is defined as

$$
\mathcal{L}(q ; \theta)=\left\langle\ln \left(\frac{p(y, z ; \theta)}{q(z)}\right)\right\rangle_{q}
$$

The variational EM algorithm is a recursive algorithm for estimating $\theta$. It consists of the two following steps at each iteration $i$ :

- Expectation (E)-step (update $q$ ):

$$
q^{\star}=\underset{q \in \mathcal{F}}{\operatorname{argmax}} \mathcal{L}\left(q ; \theta_{i-1}\right)
$$

- Maximization (E)-step (update $\theta$ ):

$$
\theta_{i}=\underset{\theta}{\operatorname{argmax}} \mathcal{L}\left(q^{\star} ; \theta\right) .
$$

In the case of multichannel HR-NMF, $\theta$ has been defined in section 2, and we define $\delta_{m}(f, t)=1$ if $y_{m}(f, t)$ is observed, otherwise $\delta_{m}(f, t)=0$ (in particular $\delta_{m}(f, t)=0 \forall(f, t) \notin[0 \ldots F-$ $1] \times[0 \ldots T-1])$, so that the complete data consists of:
- the set $y$ of observed variables $y_{m}(f, t)$ for $m \in[0 \ldots M-1]$ and for all $f$ and $t$ such that $\delta_{m}(f, t)=1$,

- the set $z$ of latent variables $z_{s}(f, t)$ for $s \in[0 \ldots S-1]$, $f \in[0 \ldots F-1], t \in\left[-Q_{z} \ldots T-1\right]$.

Besides, we use a mean field approximation [11]: $\mathcal{F}$ is defined as the set of PDFs which can be factorized in the form

$$
q(z)=\prod_{s=0}^{S-1} \prod_{f=0}^{F-1} \prod_{t=-Q_{z}}^{T-1} q_{s f t}\left(z_{s}(f, t)\right) .
$$

With this particular factorization of $q(z)$, the solution of (3) is such that each PDF $q_{s f t}$ is Gaussian: $z_{s}(f, t) \sim \mathcal{N}_{\mathbb{F}}\left(\overline{z_{s}}(f, t), \gamma_{z_{s}}(f, t)\right)$.

\subsection{Variational free energy}

Let $\alpha=1$ if $\mathbb{F}=\mathbb{C}$, and $\alpha=2$ if $\mathbb{F}=\mathbb{R}$. The joint probability distribution of the complete data can be written as

$$
\begin{aligned}
& -\alpha \ln (p(y, z ; \theta))=\left(D+S F\left(T+Q_{z}\right)\right) \ln (\alpha \pi) \\
& +D \ln \left(\sigma_{y}^{2}\right)+\frac{1}{\sigma_{y}^{2}} \sum_{m=0}^{M-1} \sum_{f=0}^{F-1} \sum_{t=0}^{T-1}\left|e_{y_{m}}(f, t)\right|^{2} \\
& +\sum_{s=0}^{S-1} \sum_{f=0}^{F-1} \sum_{t=-Q_{z}}^{-1}-\ln \left(\rho_{s}(f, t)\right)+\rho_{s}(f, t)\left|z_{s}(f, t)-\mu_{s}(f, t)\right|^{2} \\
& +\sum_{s=0}^{S-1} \sum_{f=0}^{F-1} \sum_{t=0}^{T-1} \ln \left(\sigma_{x_{s}}^{2}(t)\right)+\frac{1}{\sigma_{x_{s}}^{2}(t)}\left|e_{x_{s}}(f, t)\right|^{2}
\end{aligned}
$$

where we have introduced

$$
\begin{aligned}
D & =\sum_{m=0}^{M-1} \sum_{f=0}^{F-1} \sum_{t=0}^{T-1} \delta_{m}(f, t) \\
I(f, t) & =\mathbb{1}_{0 \leq f<F} \mathbb{1}_{0 \leq t<T} \\
e_{y_{m}}(f, t) & =\delta_{m}(f, t)\left(y_{m}(f, t)-\sum_{s=0}^{S-1} y_{m s}(f, t)\right) \\
e_{x_{s}}(f, t) & =I(f, t)\left(\sum_{\tau=0}^{Q_{a}} a_{s}(f, \tau) z_{s}(f, t-\tau)\right)
\end{aligned}
$$

Then the variational free energy defined in (2) becomes

$$
\begin{aligned}
& -\alpha \mathcal{L}(q ; \theta)=D \ln (\alpha \pi)-S F\left(T+Q_{z}\right) \\
& +D \ln \left(\sigma_{y}^{2}\right)+\sum_{m=0}^{M-1} \sum_{f=0}^{F-1} \sum_{t=0}^{T-1} \frac{\gamma_{e_{m}}(f, t)+\left|\bar{e}_{y_{m}}(f, t)\right|^{2}}{\sigma_{y}^{2}} \\
& +\sum_{s=0} \sum_{f=0}^{F-1} \sum_{t=-Q_{z}}^{-1}-\ln \left(\rho_{s}(f, t) \gamma_{z_{s}}(f, t)\right) \\
& +\rho_{s}(f, t)\left(\gamma_{z_{s}}(f, t)+\left|\bar{z}_{s}(f, t)-\mu_{s}(f, t)\right|^{2}\right) \\
& +\sum_{s=0}^{S-1} \sum_{f=0}^{F-1} \sum_{t=0}^{T-1} \ln \left(\sigma_{x_{s}}^{2}(t)\right)-\ln \left(\gamma_{z_{s}}(f, t)\right)+\frac{\gamma_{x_{s}}(f, t)+\left|\bar{e}_{x_{s}}(f, t)\right|^{2}}{\sigma_{x_{s}}^{2}(t)}
\end{aligned}
$$

where $\forall f \in[0 \ldots F-1], \forall t \in[0 \ldots T-1]$,

$$
\begin{aligned}
& \gamma_{e_{y m}}(f, t)=\delta_{m}(f, t) \sum_{s=0}^{S-1} \sum_{\varphi=-P_{b}}^{P_{b}} \sum_{\tau=0}^{Q_{b}}\left|b_{m s}(f, \varphi, \tau)\right|^{2} \gamma_{z_{s}}(f-\varphi, t-\tau) \\
& \bar{e}_{y_{m}}(f, t)=\delta_{m}(f, t)\left(y_{m}(f, t)-\sum_{s=0}^{S-1} \bar{y}_{m s}(f, t)\right) \\
& \bar{y}_{m s}(f, t)=\sum_{\varphi=-P_{b}}^{P_{b}} \sum_{\tau=0}^{Q_{b}} b_{m s}(f, \varphi, \tau) \bar{z}_{s}(f-\varphi, t-\tau) \\
& \gamma_{x_{s}}(f, t)=I(f, t)\left(\sum_{\tau=0}^{Q_{a}}\left|a_{s}(f, \tau)\right|^{2} \gamma_{z_{s}}(f, t-\tau)\right) \\
& \bar{e}_{x_{s}}(f, t)=I(f, t)\left(\sum_{\tau=0}^{Q_{a}} a_{s}(f, \tau) \bar{z}_{s}(f, t-\tau)\right)
\end{aligned}
$$




\subsection{Variational EM algorithm for multichannel HR-NMF}

In this section, the maximizations in equations (3) and (4) are performed for each scalar parameter in turn. The resulting dominant complexity of each iteration of this variational EM algorithm is $4 M F S T \Delta F \Delta T$, where $\Delta f=1+2 P_{b}$, and $\Delta t=1+Q_{z}$. However we highlight a possible parallel implementation, by making a difference between parfor loops which can be implemented in parallel, and for loops which have to be implemented sequentially.

\subsubsection{E-step}

For all $s \in[0 \ldots S-1], f \in[0 \ldots F-1], t \notin\left[-Q_{z},-1\right]$, let $\rho_{s}(f, t)=0$. Considering the mean field approximation (5), the Estep defined in (3) leads to the updates described in Table 1 (where $*$ denotes complex conjugation). Note that the updates of $\gamma_{z_{s}}(f, t)$ and $\bar{z}_{s}(f, t)$ have to be processed sequentially.

\subsubsection{M-step}

The M-step defined in (4) leads to the updates described in Table 2. The updates of the four parameters can be processed in parallel.

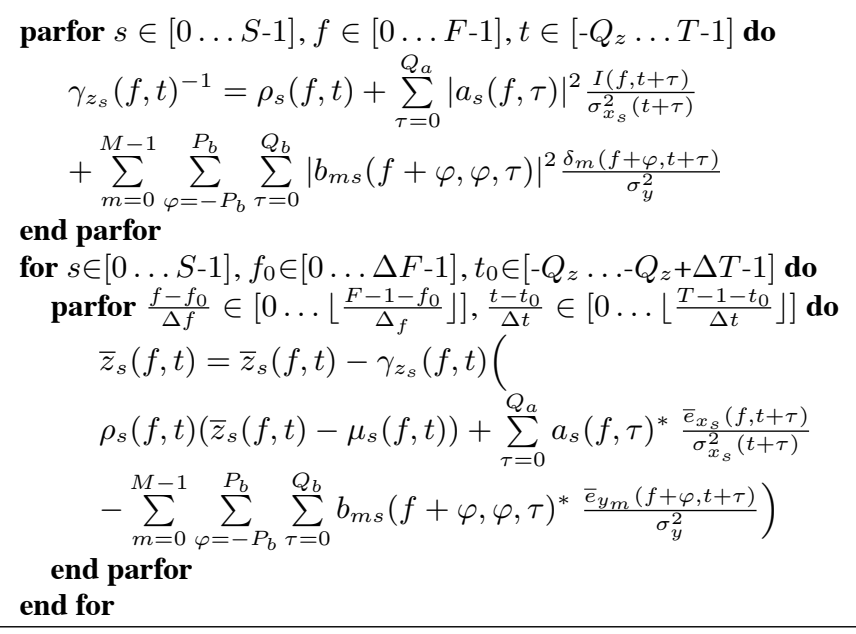

Table 1: E-step

\section{SIMULATION RESULTS}

In this section, we present a basic proof of concept of the proposed multichannel HR-NMF model. The following experiments deal with a unique source $(S=1)$ formed of a real piano sound sampled at $11025 \mathrm{~Hz}$. A stereophonic signal $(M=2)$ of length $1.25 \mathrm{~ms}$ has been synthesized by filtering the monophonic recording of a $\mathrm{C} 3$ piano note with two simulated room impulse responses. The TF representation $y_{m}(f, t)$ of this signal has then been computed by applying a critically sampled perfect reconstruction cosine modulated filterbank $(\mathbb{F}=\mathbb{R})$ with $F=201$ frequency bands, involving filters of length $8 F=1608$ samples. The resulting TF representation, of dimensions $F \times T$ with $T=77$, is displayed in Figure 1. In this configuration, the interchannel time difference is greater than the length of time frames, which suggests that the length $Q_{b}$ of filters $b_{m s}(f, \varphi, \tau)$ should be chosen greater than zero.

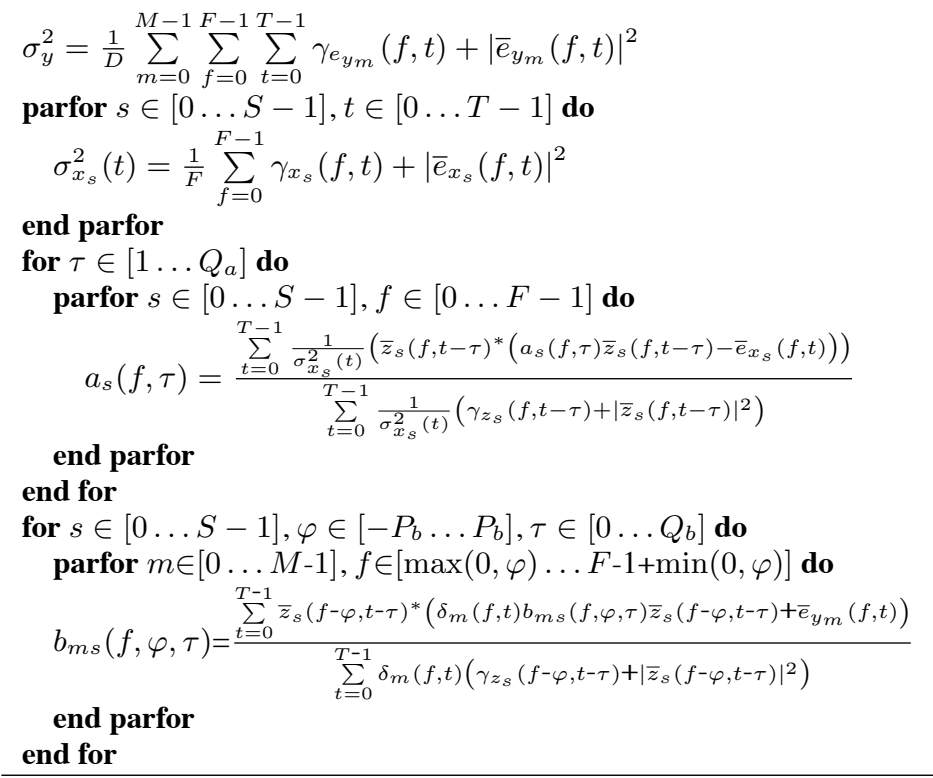

Table 2: M-step
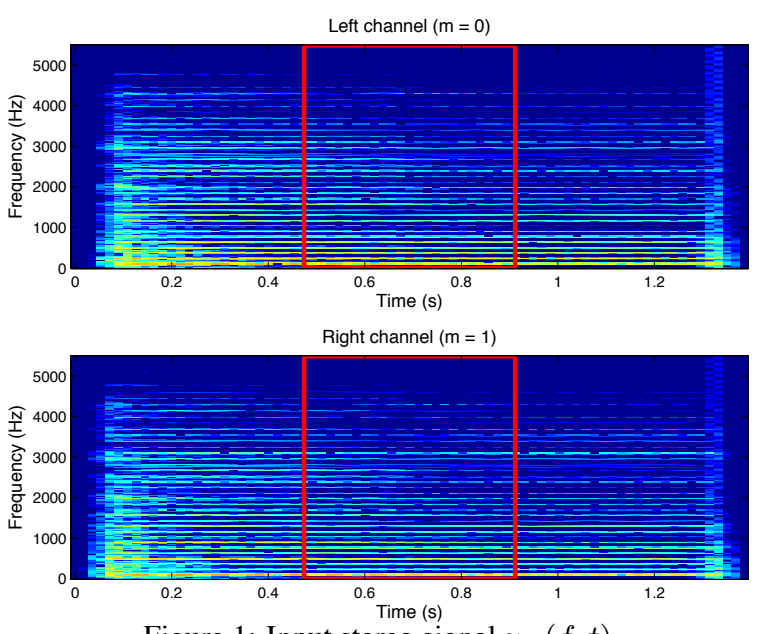

Figure 1: Input stereo signal $y_{m}(f, t)$.

In the following experiments, we have set $\mu_{s}(f, t)=0$ and $\rho_{s}(f, t)=10^{5}$ (which means that the observed sound is preceded by silence). The variational EM algorithm is initialized with $\bar{z}_{s}(f, t)=0, \gamma_{z_{s}}(f, t)=\sigma_{y}^{2}=\sigma_{x_{s}}^{2}(t)=1, a_{s}(f, \tau)=\mathbb{1}_{\{\tau=0\}}$, and $b_{m s}(f, \varphi, \tau)=\mathbb{1}_{\{\varphi=0, \tau=0\}}$. In order to illustrate the capability of the multichannel HR-NMF model to synthesize realistic audio data, we address the case of missing observations: all TF points within the red frame in Figure 1 are unobserved $\left(\delta_{m}(f, t)=0\right.$ $\forall t \in[26 \ldots 50]$, and $\delta_{m}(f, t)=1$ for all other $t$ in $\left.[0 \ldots T-1]\right)$. In each experiment, 100 iterations of the algorithm are performed, and the restored signal is returned as $\bar{y}_{m s}(f, t)$.

In the first experiment, a multichannel HR-NMF with $Q_{a}=$ $Q_{b}=P_{b}=0$ is estimated. As highlighted in section 2, this is equivalent to modeling the two channels by two IS-NMF models [4] having distinct spectral atoms and sharing the same temporal activation, or by a multichannel NMF of rank 1 [8]. The resulting TF representation $\bar{y}_{m s}(f, t)$ is displayed in Figure 2 . It can be noticed that 
wherever $y_{m}(f, t)$ is observed $\left(\delta_{m}(f, t)=1\right), \bar{y}_{m s}(f, t)$ does not accurately fit $y_{m}(f, t)$, because the length $Q_{b}$ of filters $b_{m s}(f, \varphi, \tau)$ has been underestimated. In other respects, the missing observations $\left(\delta_{m}(f, t)=0\right)$ could not be restored $\left(\bar{y}_{m s}(f, t)\right.$ is zero inside the red frame), because the correlations between contiguous TF coefficients in $y_{m}(f, t)$ have not been taken into account.

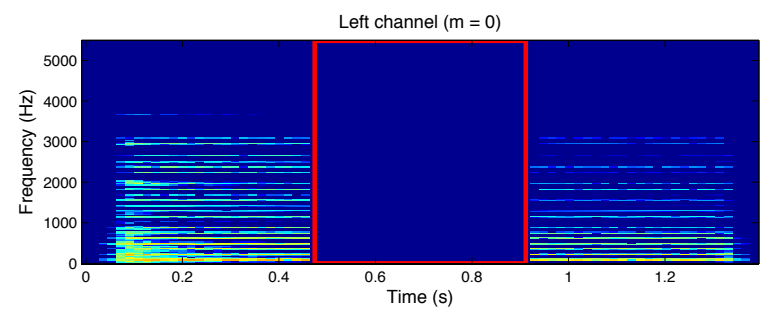
Right channel $(m=1)$

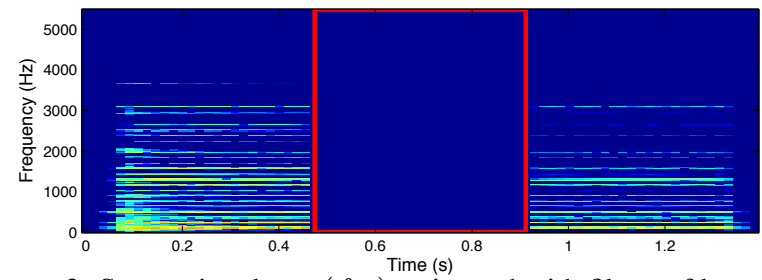

Figure 2: Stereo signal $\bar{y}_{m s}(f, t)$ estimated with filters of length 1 .

In the second experiment, a multichannel HR-NMF model with $Q_{a}=2, Q_{b}=3$, and $P_{b}=1$ is estimated. The resulting TF representation $\bar{y}_{m s}(f, t)$ is displayed in Figure 3. It can be noticed that wherever $y_{m}(f, t)$ is observed, $\bar{y}_{m s}(f, t)$ better fits $y_{m}(f, t)$. Besides, the missing observations have been correctly estimated.
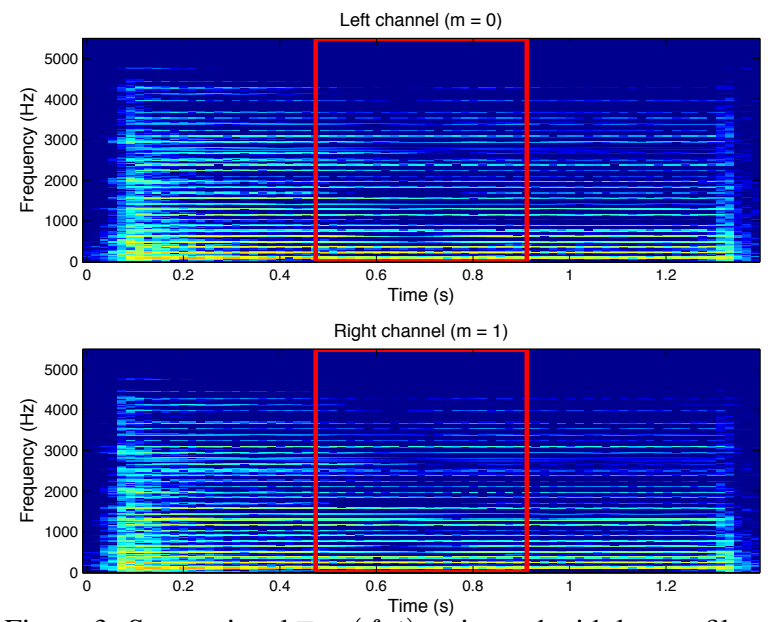

Figure 3: Stereo signal $\bar{y}_{m s}(f, t)$ estimated with longer filters.

\section{CONCLUSIONS}

In this paper, the HR-NMF model $[5,6]$ has been extended to multichannel signals and to convolutive mixtures. The new multichannel HR-NMF model accurately represents convolution in the TF domain [9], and also takes the correlations over frequencies into account. In order to estimate this model from real audio data, a variational EM algorithm has been proposed, whose novelties compared to [10] include a reduced computational complexity and a parallel implementation. This algorithm has been successfully applied to a stereophonic piano signal, and has been capable of accurately modelling reverberation and restoring missing observations.

In future work, the sparsity of the model parameters in the TF domain could be enforced by using sparse Baysian learning [12]. Some other desirable properties such as harmonicity and temporal or spectral smoothness could also be enforced by introducing some prior distributions of the parameters. Similarly to the high spectral resolution, a high temporal resolution could be achieved by extending the model as proposed in [9]. Other Bayesian estimation techniques such as Markov chain Monte Carlo (MCMC) methods and message passing algorithms [11] might prove more effective than the variational EM algorithm. Lastly, the proposed approach could be used in a variety of applications, such as source separation, source coding, audio inpainting, and automatic music transcription.

\section{REFERENCES}

[1] M.N. Schmidt and H. Laurberg, "Non-negative matrix factorization with Gaussian process priors," Computational Intelligence and Neuroscience, vol. 2008, pp. 1-10, 2008, article ID 361705 .

[2] P. Smaragdis, Blind Speech Separation. Springer, 2007, ch. Probabilistic decompositions of spectra for sound separation, pp. 365-386.

[3] T. Virtanen, A. Cemgil, and S. Godsill, "Bayesian extensions to non-negative matrix factorisation for audio signal modelling," in Proc. of IEEE ICASSP, Las Vegas, Nevada, USA, Apr. 2008, pp. 1825-1828.

[4] C. Févotte, N. Bertin, and J.-L. Durrieu, "Nonnegative matrix factorization with the Itakura-Saito divergence. With application to music analysis," Neural Computation, vol. 21, no. 3, pp. 793-830, Mar. 2009.

[5] R. Badeau, "Gaussian modeling of mixtures of non-stationary signals in the time-frequency domain (HR-NMF)," in Proc. of WASPAA, New York, USA, Oct. 2011, pp. 253-256.

[6] - - "High resolution NMF for modeling mixtures of nonstationary signals in the time-frequency domain," Télécom ParisTech, Paris, France, Tech. Rep. 2012D004, July 2012.

[7] M. H. Hayes, Statistical Digital Signal Processing And Modeling. Wiley, Aug. 2009.

[8] A. Ozerov and C. Févotte, "Multichannel nonnegative matrix factorization in convolutive mixtures for audio source separation," IEEE Trans. Audio, Speech, Language Process., vol. 18, no. 3, pp. 550-563, Mar. 2010.

[9] R. Badeau and M. D. Plumbley, "Probabilistic time-frequency source-filter decomposition of non-stationary signals," in Proc. of EUSIPCO, Marrakech, Morocco, Sept. 2013, submitted for publication.

[10] R. Badeau and A. Drémeau, "Variational Bayesian EM algorithm for modeling mixtures of non-stationary signals in the time-frequency domain (HR-NMF)," in Proc. of IEEE ICASSP, Vancouver, Canada, May 2013.

[11] D. J. MacKay, Information Theory, Inference, and Learning Algorithms. Cambridge University Press, 2003.

[12] D. P. Wipf and B. D. Rao, "Sparse bayesian learning for basis selection," IEEE Trans. Signal Process., vol. 52, no. 8, pp. 2153-2154, Aug. 2004. 